\title{
Evidence-based Medicine in Cardiac Imaging
}

\author{
Maseeh uz Zaman and Nosheen Fatima
}

The term evidence-based medicine (EBM) was first coined by $\mathrm{Dr}$ Gordon Guyatt, a physician and epidemiologist at McMaster University, Canada. ${ }^{1}$ He defined EBM as "medicine practised on the basis of evidence". Soon after the publication of this single author article in 1991,1 a group of physicians and epidemiologists launched a campaign by the name of EBM Movement. ${ }^{2}$ They emphasised upon greater reliance on up-to-date and published research; especially, randomised control trials (RCT) and systematic reviews (SR). Similarly, evidence based practice is provision of best possible care to patients by considering their preference, clinical setting and available best evidence by the trained healthcare providers. The 28-year journey of EBM is marked by many success stories. First, it has successfully captured the agenda that the scientists and physicians will decide the future of medicine; and indeed this is a great achievement. ${ }^{3}$ Next is foundation of Cochrane Collaboration in 1993 in Oxford, United Kingdom by National Health Services (NHS) in which more than 40,000 volunteers are associated with it, who are producing more than 400 systematic reviews of clinical trials annually. ${ }^{4}$ These systematic reviews are the bases of formulation of clinical guidelines which are regularly published by professional societies for good clinical practice and decision-making. These systematic reviews also fuel the formulation of appropriate use criteria (AUC) for judicious use of the procedures and devices by various professional societies. ${ }^{5}$ EBM has recently launched a campaign demanding pharmaceutical industry to disclose the data of clinical trials which have been funded by them. This agenda has been warmly welcomed and supported by World Health Organization (WHO) and European Union (EU).6,7 Recently, EBM has coined a terminology "overdiagnosis", not leading to reduced mortality associated with a particular disease. ${ }^{8}$ This has been observed for the significantly increased incidence of thyroid and prostate cancers due to overwhelming use of fineneedle aspiration in thyroid nodule and serum PSA without appreciable decline in cancer related mortality. ${ }^{8}$ It is imperative to improve clinical outcome with respect

Department of Radiology, The Aga Khan University Hospital, Karachi, Pakistan

Correspondence: Prof. Maseeh uz Zaman, Section of NM \& PET/CT Imaging Services, Department of Radiology,

The Aga Khan University Hospital, Karachi, Pakistan

E-mail:maseeh.uzzaman@aku.edu

Received: December 24, 2018; Revised: January 19, 2019; Accepted: March 08, 2019 to clinical need and cost; and hopefully, EBM campaign will produce a positive impact.

There are four basic elements of EBM. ${ }^{9}$ First, a clinical question is formulated which has four pertinent information, i.e. Patient, Intervention, Comparison and Outcome (PICO).10 A PICO question could be like "Dobutamine stress echocardiography has better prognostic value than myocardial perfusion imaging for patients with acute myocardial infarction". For this PICO question, best available evidences are searched from available published data in the form of randomised control trials as these have lower odds of bias; and studies with good patients' population, reduce the chances of error. Then these published data are validated internally and externally. Finally, the results extracted from validated data are applied in clinical practice in conjunction with clinical expertise and patients' preferences. Gathered information about a PICO question is than issued by the professional society or body in the form of clinical guidelines. ${ }^{9}$ Usually, these clinical guidelines have recommendations for practising physicians with weightage based on evidences.

The recommendations in clinical guidelines are usually classified as Class I: having evidence and/or general agreement about the usefulness / efficacy of a procedure or treatment; Class II: having conflicting evidence about the usefulness and efficacy of the particular procedure or treatment; Class III: there is evidence of a general agreement that particular procedure is not useful and effective or may be harmful. These recommendations are supported by evidence which is further classified on the basis of its origin. Evidence drawn from multiple randomised trials are considered strong (Level A) and those drawn from single randomised trial or non-randomised trials is considered fair (Level B). Evidence which is only based on consensus opinions of experts and not having support of published data are considered weak (Level C). ${ }^{11}$

Similarly, this validated evidence is also used for Appropriate Use Criteria (AUC) for certain clinical procedures and devices, which are released by professional societies. A procedure is considered appropriate for which the expected health benefits exceed expected risk by a wide margin. ${ }^{5}$ Contrary to clinical guidelines, AUC are often consensus of experts' opinion with a weak scientific basis. There is also a possibility that if a physician feels that he or she would be judged on the basis of $A U C$, it is quite possible that the physician may use a diagnosis that fits with $A \cup C$ and 
not the real one for that required procedure. However, it is also important to understand that there is significant discordance between AUC published for nuclear myocardial perfusion imaging from different societies. ${ }^{12}$

It can be argued whether we have EBM in cardiac imaging? A recently published study revealed that medicare billing for medical imaging had increased from $\$ 7$ billion in 2000 to $\$ 14$ billion in 2005.13 Furthermore, approximately one-third of billing amount involved cardiovascular medical imaging which were adopted at an extraordinary cost with insufficient evidence of benefits to patients. ${ }^{13}$ Another study published in 2009 revealed that $30-50 \%$ of cardiac procedures performed were appropriate and basic reasons were market messages, high patient demand and defensive medicine, leading to the vicious circle of the so-called Ulysses syndrome. ${ }^{14}$ However, the most important concern has been raised by American Heart Association (AHA) in its scientific statement published in 2014. This states that the radiation exposure to US population from medical imaging has increased six-fold since 1980 and over $40 \%$ of this has been contributed by the cardiovascular imaging and intervention. ${ }^{15}$ This important fact indeed draws attention to sensitize the medical community about the importance of EBM in cardiac imaging, particularly to minimize the lifetime attributable risk for radiation associated cancer in exposees.

It is imperative to understand the possible obstacles in implementation of EBM in cardiac imaging. The most important obstacle is over-reliance on the reliability of clinical trials and systematic reviews. For example, two systematic reviews about the impact of beta-blockers on mortality in acute myocardial infarction had totally different outcomes; one favoured and the other denied the role of beta blocker in reducing mortality. ${ }^{3}$ Overreliance on statistical significance of results of published data is another considerable obstacle. Statistical methods used in analysis need to be carefully studied as inappropriate use could make a non-significant $p$-value to a statistically significant one. Therefore, while reading published studies, one must consider clinical as well as statistical significance of data mentioned in a clinical trial or systematic review. It is also not uncommon to have incomplete and conflicting recommendations by professional societies, that clinicians feel to be well away from real practice. It is also important to understand that the non-availability of certain equipment and patients' preferences are also important hurdles in implementation of EBM in cardiac imaging. Lastly, the financial aspect, associated with cardiac imaging like reimbursement by insurance companies or financial malpractice among referring physicians and cardiac laboratories, is also an undeniable obstacle. ${ }^{3}$

For effective implementation of EBM in a cardiac imaging, a multifaceted approach is considered most appropriate.
We must acquire clinical information from educational materials, like review articles and meta-analyses, with recently published guidelines and AUC by the relevant professional societies. We must educate staff and physicians about AUC for specific clinical questions. Studies have shown considerable variation in raters at different levels of training in identifying inappropriate nuclear cardiac imaging procedures. ${ }^{16}$ With appropriate training, improvements have been observed for appropriate use of transthoracic echocardiography (TTE) and CT angiography (CTA), but not for trans-esophageal echocardiography (TEE) and stress imaging. 17 While performing cardiac imaging, one must perform complete evaluation and documentation that procedure meets relevant AUC criteria. In institute having Picture Archiving and Communication Systems (PACS), Computerized Physician Order Entry (CPOE) and frequent reminders about the importance of appropriate cardiac imaging procedures, can be installed. When physician enters an order for a cardiac imaging procedure, the software asks few clinical queries pertinent to AUC for that particular imaging. Effective communication among staff, nurses and referring physicians can also help by convincing the referring physician about an appropriate imaging against his or her patient's clinical query. To address financial issues, one can link the reimbursement with practice of EBM as per clinical guidelines and AUC. American Congress has passed a bill in 2014: "As of 2017, claims without documentation that AUC criteria were consulted, will not be paid by medicare". ${ }^{18}$ It also states that documentation is the responsibility of the referring physician but the payment would be at risk for the performing physician and this has indeed created conflict between them.

Evidence-based medicine has become an explicit element of medical professionalism, rendering to launch a successful campaign that physicians and scientists will decide the future of medicine. It has introduced culture of clinical guidelines and AUC for better clinical decisionmaking in cardiology and other faculties of medicine. However, it has also contributed tension between the different sectors of healthcare. Despite these obstacles, there is optimism for EBM future in cardiac imaging as it would reduce probability of radiation-associated cancers by curtailing inappropriate and unjustified radiationbased procedures. To achieve this aim, there is a unanimous call for more evidence to support evidencebased cardiac imaging in future. It is believed that "lack of evidence does not mean absence of evidence."

\section{REFERENCES}

1. Guyatt GH. Evidence-based medicine. ACP J Club 1991; 114: A16.

2. Evidence-based medicine working group. Evidence-based medicine: A new approach to teaching the practice of medicine. JAMA 1992; 268:2420-5. 
3. Sheridan DJ, Julian DG. Achievements and limitations of evidence-based medicine. J Am Coll Cardiol 2016; 68:204-13.

4. Cochrane library oversight committee. Measuring the performance of the Cochrane library. Cochrane Database Syst Rev 2012; 12:ED000048.

5. Armstrong W, Eagle KA. "Appropriate use criteria in echocardiography: Is no change the same as no benefit?". JAMA Intern Med 2013; 173:1609-10.

6. Moorthy VS, Karam G, Vannice KS. Rationale for WHO's new position calling for prompt reporting and public disclosure of interventional clinical trial results. PLoS Med 2015; 12: e1001819.

7. La Santos Quintana A. PMp: EU's clinical trials regulation 'a step in right direction' parliament magazine, 2014. Available at: https://www. theparliamentmagazine.eu/articles/sponsored_ article/pm-eu\%E2\%80\%99s-clinical-trials-regulation$\%$ E2\%80\%98-step-right-direction\%E2\%80\%99. [Accessed 22.12.2018].

8. Welch HG, Black WC. Overdiagnosis in cancer. J Natl Cancer Inst 2010; 102:605-13.

9. Sackett DL, Straus SE, Richardson WS. Evidence-based medicine: How to practice and teach EBM. ed 2nd, Churchill Livingstone, Edinburgh 2000.

10. Richardson WS, Wilson MC, Nishikawa J, Hayward RS. The well-built clinical question: A key to evidence-based decisions. ACP J Club 1995; 123:A12.
11. Budoff MJ, Achenbach S, Blumenthal RS, Carr JF, Goldin JG, Greenland $\mathrm{P}$, et al. Assessment of coronary artery disease by cardiac computed tomography. Circulation 2006; 114:1761-91.

12. Winchester DE, Wolinsky D, Beyth RJ, Shaw LJ. Discordance between appropriate use criteria for nuclear myocardial perfusion imaging from different specialty societies: A potential concern for health policy. JAMA Cardiol 2016; 1:207-10.

13. Farmer SA. CV testing practices for incident HF vary widely across hospitals. JACC Cardiovasc Imaging 2014; 7:690-700.

14. Picano $\mathrm{E}$. The risks of inappropriateness in cardiac imaging. Int J Environ Res Public Health 2009; 6:1649-64.

15. Reza Fazel R, Gerber TC, Balter S, Brenner DJ, Carr JJ, Cerqueira MD, et al. Approaches to enhancing radiation safety in cardiovascular imaging. Circulation 2014; 130:1730-48.

16. Ye S, Rabbani LR, Kelly CR, Kelly MR, Lewis M, Paz Y, et al. Can physicians identify inappropriate nuclear stress tests? Examination of inter-rater reliability for the 2009 appropriate use criteria for radionuclide imaging. Circ Cardiovasc Qual Outcomes 2015; 8:23-9.

17. Fonseca R, Negishi K, Otahal P, Marwick TH. Temporal changes in appropriateness of cardiac imaging. J Am Coll Cardiol 2015; 65:763-73.

18. http://www.cardiovascularbusiness.com/topics/imaging/accexamines-impact-medicare-changes-imaging 